\title{
Interaction-induced topological charge pump
}

\author{
Yoshihito Kuno $\odot$ and Yasuhiro Hatsugai $\odot$ \\ Department of Physics, University of Tsukuba, Tsukuba, Ibaraki 305-8571, Japan
}

(Received 27 July 2020; accepted 9 October 2020; published 30 October 2020)

\begin{abstract}
Based on a topological transition of the symmetry-protected topological phase (SPT), an interaction-induced topological charge pump (iTCP) is proposed with the symmetry-breaking parameter as a synthetic dimension. It implies that the phase boundary of the SPT is a topological obstruction, although iTCP and the gap closing singularity are stable for symmetry-breaking perturbations. As for the iTCP, an interaction is essential since the pumped charge is trivial for a noninteracting system. We have confirmed the bulk-edge correspondence for this iTCP using density matrix renormalization group for the Rice-Mele model with nearest-neighbor interactions. As for a realization in optical lattices, an interaction sweeping pump protocol is proposed as well.
\end{abstract}

DOI: 10.1103/PhysRevResearch.2.042024

Introduction. A topological charge pump (TCP) [1] is a fundamental topological phenomenon, which includes the essence of topological condensed matter [2,3]. Since the TCP was first proposed by Thouless about four decades ago [1], it has been rarely verified experimentally. However, recent experimental systems, such as cold atoms in an optical lattice [4-6] and photonic crystals [7], have enabled the realization of TCP. Experimental realizations have made the study of TCP one of the most active current topics. In particular, motivated by the significant controllability in recent cold atom and photonic systems $[8,9]$, TCP has been focused theoretically from a new point of view. Although the roles of the edge states have yet to be discussed as for TCP, the bulk-edge correspondence (BEC) of TCP of the noninteracting fermions was reconsidered in Ref. [10].

Unlike various topological phenomena where the bulk topological number is hidden and the edge states are physical observables, the physical observable of TCP is a bulk current and the edge states are hidden (never pumped in a finite speed pump). We here establish BEC of TCP for the interacting system after a general proposal of TCP based on the symmetry-protected topological (SPT) phase transition.

So far, motivated by the recent experimental success of TCP, various theoretical works of TCP have been reported. TCP and a topological Mott phase related to TCP with a Hubbard interaction [11,12], interacting bosonic systems [13-17], and magnon pumps [18] have been discussed. Further, randomness and nonadiabaticity have been studied in Refs. [19-23]. In this Rapid Communication, we propose an interaction-induced topological charge pump (iTCP) based on the general scheme. Since TCP is independent of any

Published by the American Physical Society under the terms of the Creative Commons Attribution 4.0 International license. Further distribution of this work must maintain attribution to the author(s) and the published article's title, journal citation, and DOI. symmetry protection, the existence of a nontrivial pump is not trivial a priori. Then mapping from a two-dimensional topologically nontrivial system such as quantum Hall states by replacing one of the physical dimensions with a time is useful $[1,24]$. We here propose another general scheme to realize nontrivial TCP (see Fig. 1).

The starting point is a one-dimensional gapped SPT phase [24-27] that is characterized by the symmetry-protected Berry phase $i \gamma=\int A$, where $A=\psi^{\dagger} d \psi$ and $\psi$ is the ground state $[24,28,29]$. The Berry connection $A$ is generally defined for a twisted boundary condition $S_{\theta}^{1}=\left\{e^{i \theta} \mid \theta \in(0,2 \pi]\right\}[30,31]$.

Here, we consider two different SPT phases $P_{1}$ and $P_{2}$ characterized by different $\gamma$ 's on a two-dimensional plane spanned by two parameters $\lambda_{1}$ and $\lambda_{2}$ where the symmetry of the SPT is preserved. We call this the "SPT plane." Then, the presence of two different SPT phases leads to a gapless phase boundary line on the SPT plane, which separates the two SPT phases $P_{1}$ and $P_{2}$ [32] [the line may end due to the appearance of symmetry-breaking phases such as a charge density wave (CDW) for large interactions].

Here, we extend the parameter space by introducing an extra (synthetic) dimension $\lambda_{3}$ that is a symmetry-breaking parameter for the SPT. In the total parameter space, we introduce a loop $\ell$ connecting the two SPT phases $P_{1}$ and $P_{2}$ without crossing the gapless phase boundary line on the SPT plane. This loop can host a nontrivial pump [33] since the phase boundary line passing through the loop acts as a topological obstruction. The loop cannot be shrunk to a single point adiabatically. Moreover, this construction implies that once the nontrivial TCP is realized, symmetry protection to realize the SPT phase can be relaxed as long as the gap along the loop remains open. This point will be discussed later.

Using this nontrivial topological structure of the extended phase space, one can construct various pump protocols. We are proposing an $\mathrm{iTCP}$ when the interaction is one of the axes on the SPT plane. We demonstrate two cases: (i) a nontrivial pump induced only by interaction sweep, and (ii) a nontrivial pump with a finite interaction, which is trivial for the noninteracting case. 


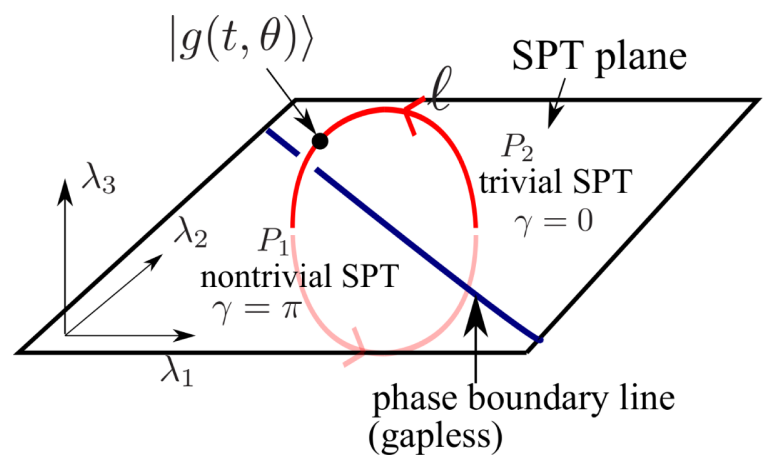

FIG. 1. A schematic of a topological pump protocol (red line) protected by a gapless phase boundary passing through the protocol loop $\ell .|g(t, \theta)\rangle$ is a ground state of the snapshot Hamiltonian $H(t, \theta)$.

Also, we have established the BEC for the iTCP. In the open system, the nontrivial $\gamma$ of the SPTs on the SPT plane implies the existence of edge states and the different SPTs have different numbers of edge states [34,35]. When the nontrivial pump loop connects two different SPT phases $P_{1}$ and $P_{2}$ with different numbers of edge states, it is expected that the pump induced by the loop can be nontrivial.

In the following, we shall demonstrate a simple example of the above proposal by considering a simple Rice-Mele (RM) model with inner-unit-cell interactions, which is very close to recent experimental systems [4,5]. We shall numerically demonstrate the iTCP and also confirm that the BEC is established for the iTCP.

Model. The model considered in this work is a generalized Rice-Mele (gRM) model with nearest-neighbor (NN) interactions,

$$
\begin{aligned}
H_{\mathrm{gRM}}= & \sum_{j=0}^{L-1}\left[-J_{j}\left(c_{j+1}^{\dagger} c_{j}+\text { H.c. }\right)+\Delta_{j} c_{j}^{\dagger} c_{j}\right. \\
& \left.+V_{j}\left(n_{j}-\frac{1}{2}\right)\left(n_{j+1}-\frac{1}{2}\right)\right],
\end{aligned}
$$

where $c_{j}^{(\dagger)}$ is a fermion annihilation (creation) operator, $n_{j}=$ $c_{j}^{\dagger} c_{j}$, and the $J_{j}, \Delta_{j}$, and $V_{j}$ terms are the hopping amplitude, on-site potential, and NN interactions, respectively. $L$ is a system size. Here, the parameters $J_{j}, \Delta_{j}$, and $V_{j}$ take different values when $j$ is even or odd. Here, let us define $\Delta_{d}=\Delta_{j \in \text { even }}-\Delta_{j \in \text { odd }}$ as a symmetry [bond center inversion (BCI) [36]] breaking parameter. Note that finite $J_{j \in \text { even }}-J_{j \in \text { odd }}$ and $V_{j \in \text { even }}-V_{j \in \text { odd }}$ respect the BCI. We set $J_{j \in \text { even }}=J_{1}$ and $J_{j \in \text { odd }}=J_{2}$. For the $V_{j}=0$ case, if we change $J_{j}$ and $\Delta_{j}$ adiabatically, the above model can be reduced to the Rice-Mele model [37], which is a standard model of TCP [38]. Hereafter we focus on the case $V_{j \in \text { even }}=V_{\text {in }}$ and $V_{j \in \text { odd }}=0$ for simplicity.

Emergence of iTCP. When $J_{1(2)}$ is real and $\Delta_{j}=0, H_{\mathrm{gRM}}$ reduces to the $\mathrm{Su}-\mathrm{Sch}$ rieffer-Heeger ( $\mathrm{SSH}$ ) model [39] with the interactions $V_{\text {in }}$, which is a lattice analog of the GrossNeveu model [27,40,41]. Due to the particle-hole $(\mathrm{PH})$ or the BCI, a gapped SPT is realized for the half-filled case. In this Rapid Communication, we mainly consider the BCI
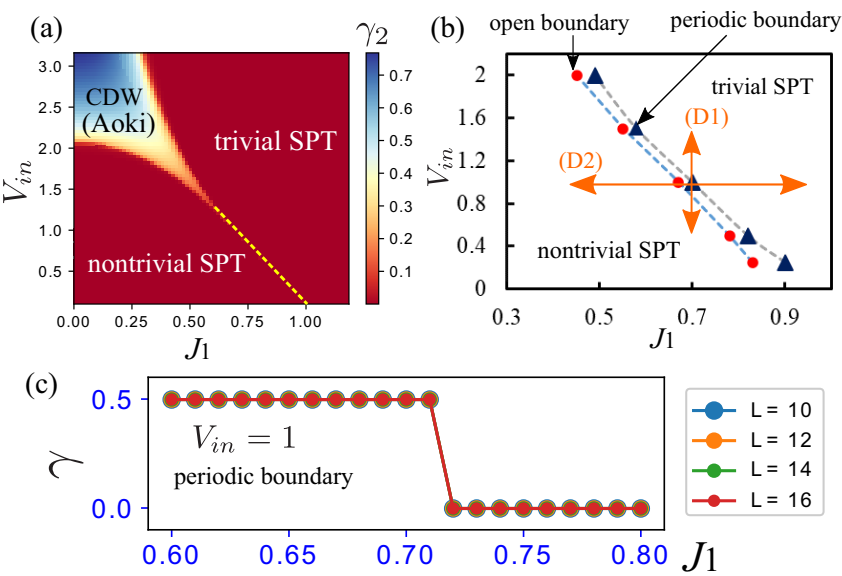

FIG. 2. (a) MF phase diagram for the interacting SSH model. $\gamma_{2}$ is the CDW order parameter (see Supplemental Material for details [42]). We set $J_{2}=1$. (b) The phase diagram for the interacting SSH model is given by the entanglement spectrum of the open system and the Berry phase associated with the twisted boundary condition. The phase boundary of the open boundary condition is fixed by the entanglement spectrum by the DMRG with $L=64$ and the Berry phase using the ED. (c) The Berry phase $\gamma$ in an $L / 2$-particle system. The sharp transition is due to the gap closing by the twist boundary condition [29].

as the symmetry to protect the SPT, including away from the half-filled situation. We set $\lambda_{1}=J_{1}$ and $\lambda_{2}=V_{\text {in }}$ to span the SPT plane. We use $\Delta_{d}$ as the symmetry-breaking parameter. In what follows, we set $J_{2}=1$.

The phase structure of this model is the starting point to realize the iTCP. A mean-field (MF) phase diagram for the SSH model with a half-filled case is shown in Fig. 2(a). The MF theory is briefly explained in Ref. [42]. The phase diagram has three phases: the nontrivial SPT, trivial SPT, and CDW (Aoki) phases. The CDW order corresponds to the Aoki phase in the high-energy physics context [27,40,43]. We do not focus on the CDW order in this Rapid Communication.

For a system without a boundary, the Berry phase $\gamma$ is quantized into $Z_{2}$, and the nontrivial SPT is characterized by $\gamma=\pi[24,44-46]$. With an open boundary condition, edge modes appear for $\gamma=\pi$ according to the BEC for the onedimensional system. By using exact diagonalization (ED), we confirmed the phase boundary line separating the nontrivial and trivial SPT phases on the $J_{1}-V_{\text {in }}$ plane, as shown in Fig. 2(b). Figure 2(c) is a typical transition behavior of $\gamma$, where a clear topological phase transition point is determined by the $Z_{2}$ Berry phase $\gamma$ without any significant system size dependence [29]. It implies that the gapless phase boundary forms a line as shown in Figs. 2(a) and 2(b).

Using the gapless phase boundary line on the $J_{1}-V_{\text {in }}$ plane as a topological obstruction, various pump protocols to exhibit the TCP can be considered (see Fig. 1). The pump protocol is specified by the loop $\ell$ parametrized by time $t$. The loop $\ell$ connects the nontrivial and trivial SPT phases without crossing the gapless phase boundary line [47]. Together with the twisted boundary condition $S^{1}$, the loop $\ell$ forms a torus $T^{2}=S^{1} \times \ell$. Then the snapshot ground state is defined on the $T^{2}$ where the twist parameter space $S^{1}$ is small in a sense that 
physical observables such as the energy are independent of $\theta$ when the system size is infinite $[48,49]$.

For concreteness, we set $\Delta_{j}(t) \equiv(-1)^{j} \Delta_{0} \sin (2 \pi t / T)$, that is, $\lambda_{3}=\Delta_{d}=2 \Delta_{0} \sin (2 \pi t / T)$, where $T$ is a period of the pump. Together with $\Delta_{j}(t)$, we can constitute a pump protocol loop $\ell$ by adiabatically varying the interaction $V_{\text {in }}$ and/or a hopping ratio $J_{1} / J_{2}$. The concrete form will be given later.

For a pump protocol loop $\ell$, the topological invariant of the iTCP is given by the Chern number in a temporal gauge as

$$
i \bar{A}_{\theta}^{(t)}(T)=C,
$$

where $\bar{A}_{\theta}^{(t)}(t) \equiv \frac{1}{2 \pi} \int_{-\pi}^{\pi} A_{\theta}^{(t)}(\theta, t) d \theta$ is a $\theta$ averaged Berry connection in the temporal gauge $A_{\theta}^{(t)}(\theta, t)$ (specified uniquely by $\left.A_{t}^{(t)}=0\right)$ as [10]

$$
A_{\theta}^{(t)}(\theta, t)=A_{\theta}(\theta, t)-\partial_{\theta} \int_{0}^{t} A_{t}(\theta, \tau) d \tau-A_{\theta}(\theta, 0),
$$

where $A_{t}(\theta, \tau)$ and $A_{\theta}(\theta, \tau)$ are Berry connections in an arbitrary gauge. Note that the Berry connection in the temporal gauge is not periodic in time even though $A_{t(\theta)}(\theta, t)$ can be periodic.

Center of mass of $i T C P$. Let us numerically demonstrate the iTCP for the system with open boundaries. We employ the density matrix renormalization group (DMRG) method [50]. For the DMRG calculations, by employing the Jordan-Wigner transformation, we map the interacting RM model into the $S=1 / 2$ spin XXZ model and use the TeNPy Library [51].

To begin with, for the system with open boundaries on the SPT plane $\Delta_{d}=0$, we have calculated the entanglement spectrum and determined the transition point on the $J_{1}-V_{\text {in }}$ plane, as shown in Fig. 2(b) [52]. The gapless phase boundary is consistent with that of the periodic case, determined by the quantized Berry phase $\gamma$.

Together with $\Delta_{j}(t)=(-1)^{j} \Delta_{0} \sin (2 \pi t / T)$, we propose the following two concrete protocols: (D1)-pump protocol loop: $\quad J_{1}=0.7, \quad J_{2}=1, \quad V_{\text {in }}(t)=1+0.5 \cos (2 \pi t / T)$, $\Delta_{0}=0.5$. (D2)-pump protocol loop: $J_{1}(t)=0.7+$ $0.25 \cos (2 \pi t / T), \quad V_{\text {in }}=1, \quad \Delta_{0}=1$. The former is an interaction sweeping protocol and the latter is a trivial protocol in the noninteracting case [see Fig. 2(b)]. Both protocol loops wrap the gapless phase boundary line.

The TCP for a finite system with open boundaries is characterized by the jump of the center of mass (c.m.) [10,11]. The c.m. is measured under the grand canonical ensemble [10], i.e., the system contact with a particle reservoir. We adapt this for the interacting system. The c.m. is given by

$$
P(t)=\sum_{j=0}^{L-1}\left\langle\Psi(t)\left|\left[\frac{j-j_{0}}{L}\right] n_{j}\right| \Psi(t)\right\rangle,
$$

where $|\Psi(t)\rangle$ is a many-body ground state at the time $t$ under the grand canonical ensemble and $j_{0}=(L-1) / 2$. The particle number $N_{e}$ of $|\Psi(t)\rangle$ is determined by the chemical potential $\mu$ as $\Delta E\left(N_{e}, t\right)<\mu$, where $\Delta E\left(N_{e}, t\right)=E\left(N_{e}+\right.$ $1, t)-E\left(N_{e}, t\right)$ and $E\left(N_{e}, t\right)=\left\langle\Psi(t)\left|H_{\mathrm{gRM}}(t)\right| \Psi(t)\right\rangle[53,54]$ (the finite chemical potential simply breaks the PH symmetry but the BCI is preserved). Figures 3(a) and 3(b) are the numerical results of $\Delta E$ around $N_{e}=L / 2$ for both (D1)-

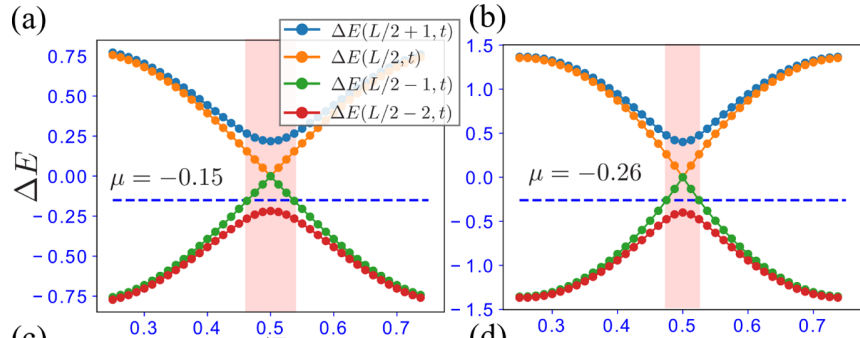

(c)

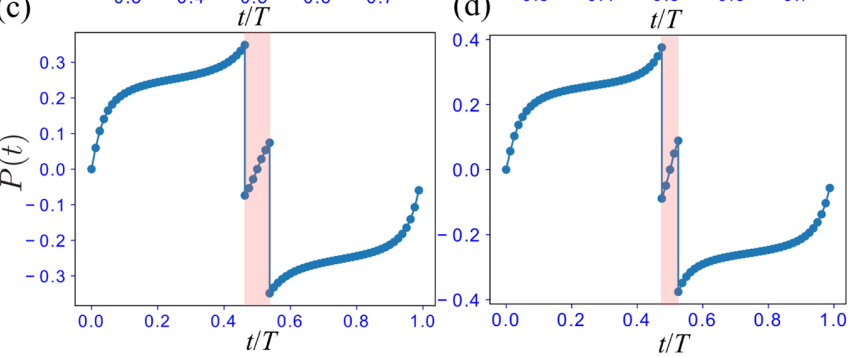

FIG. 3. Excitation spectrum $\Delta E$ : (a) (D1)-pump protocol. (b) (D2)-pump protocol. The behavior of the c.m. with fixed $\mu$ : (c) (D1)-pump protocol with $\mu=-0.15$. (d) (D2)-pump protocol with $\mu=-0.26$. In the red shaded area, the system includes $L / 2-1$ particles, and in other regions, $L / 2$ particles. For all data, $L=64$.

and (D2)-pump protocols. We find clear in-gap states for a finite $V_{\text {in }}$. It implies the number of particles that satisfies $\Delta E\left(N_{e}\right)>\mu$ changes. This induces the gap closing for the grand canonical Hamiltonian $H_{\mathrm{gRM}}-\mu N$. This gap closing is due to the edge states (as shown in later). Although it breaks the adiabaticity, it does not affect any experimental observables since the edge states are never pumped in a realistic finite speed pump.

The topological invariant $I$ of the system with boundaries is a sum of the jumps of c.m. as [10]

$$
\left.\sum_{t_{i}} P(t)\right|_{t_{i}-0} ^{t_{i}+0} \equiv \sum \Delta P\left(t_{i}\right) \stackrel{L \rightarrow \infty}{\longrightarrow}-I .
$$

The BEC implies $I=C[10]$, which we will numerically confirm for an interacting case later.

We set the chemical potential $\mu$ and determine the system particle number $N_{e}$ each time. Figures 3(c) and 3(d) are the results of the behavior of the c.m. Here, by the DMRG simulations, we find that for both (D1)- and (D2)-pump protocols, when the energy of the in-gap state is equal to the chemical potential, the c.m. jumps. The jump of $\Delta P\left(t_{i}\right)$ is associated with the change of the total number of particles. It can be attributed to the edge state (localized gapless mode). Actually, as displayed in Figs. 4(a) and 4(b), the in-gap states are left/right edge modes. When the total number of particles is changed, the edge states induce the change of the density distribution near the boundaries. It induces the single jump $\Delta P\left(t_{i}\right)$ for the c.m.

Furthermore, as shown in Fig. 4(c), for the (D1)-pump protocol, we have confirmed the convergence that as the system size increases, the total sum of the jump approaches an integer value $I: \sum_{t_{i}} \Delta P\left(t_{i}\right) \stackrel{L \rightarrow \infty}{\longrightarrow}-I$. Here, $I$ is an integer. This is the topological nature of the TCP. From these facts, with open boundaries and interactions, the (D1)- and (D2)-pump 


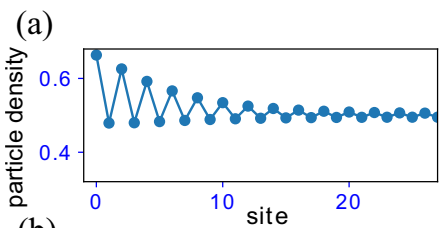

(b)
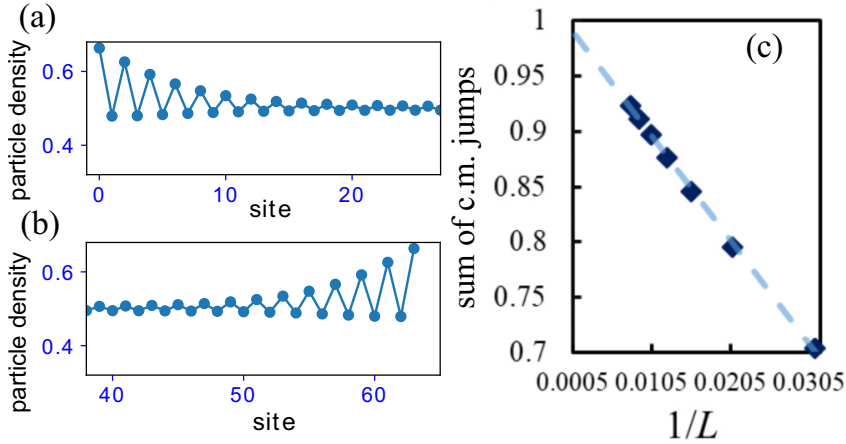

FIG. 4. The density distribution including the left and right edge state at $t / T=0.49865$ (a) and 0.50183 (b) in (D1)-pump protocol. The density distribution is defined by $\left\langle\Psi(L / 2)\left|n_{j}\right| \Psi(L / 2)\right\rangle$, where $|\Psi(L / 2)\rangle$ is a ground state with $L / 2$ particles. The value of c.m. is \pm 0.42287 . The system size is $L=64$. (c) System size dependence of the total jump of the c.m. with $\mu=-0.15$. Each of the jumps of the c.m. are induced by changing the particle number and occur at $t / T=0.46272$ and 0.53776 .

protocols exhibit the iTCP. The pumped charge is carried by the bulk even with boundaries, although its quantization is clear by interpreting the jump in the c.m. due to the edge states. This is the BEC for the TCP.

Many-body Chern number and bulk-edge correspondence. The c.m. obtained in Figs. 3(c) and 3(d) implies the presence of the TCP in the bulk. To verify it, by using the ED, we calculated the many-body Chern number $C$ for the periodic system. Numerically, $C$ is calculated by a method [55]. Focusing on the (D1) protocol, we calculated $C$ as varying the parameter $J_{1}$. The result is shown in Fig. 5(b). At $J_{1}=0.7$ we see $C=1$. The (D1)-pump protocol indicates the presence of the iTCP in the bulk. Accordingly, from the result in Fig. 3(c), $C=I$ is verified, i.e., the BEC is confirmed in the iTCP. We have also analyze the system by the MF approximation [42] that is consistent with that of the DMRG and the ED for the relative small system size. In Fig. 5(b), we also find that as increasing $J_{1}$, a topological phase transition occurs where $C$ suddenly changes from one to zero. This is because the pump protocol no longer wraps the topological obstruction [see Fig. 5(a)].

Stability of the TCP. The iTCP is stable for any finite symmetry-breaking perturbation for as long as the gap remains open. As an example of the symmetry-breaking perturbation, let us introduce an on-site potential: $\delta V=$ $\sum_{j \in \text { even }} w_{0} n_{j}$. Since $\delta V$ breaks the PH and BCI symmetries, it breaks quantization of the Berry phases and the SPT is lost. Then, what happens to the Chern number $C$ when the pump protocol loop is made smaller under a finite $\delta V$ ? To this end, we introduce a parameter $R$ to control the size of the protocol loop in the (D1)-pump protocol: $J_{1}=0.7, J_{2}=$ $1, V_{\text {in }}=1+R \cos (2 \pi t / T), \quad \Delta_{j}=(-1)^{j}(R / 2) \sin (2 \pi t / T)$. The phase diagram of $C$ on the $w_{0}-R$ plane is shown

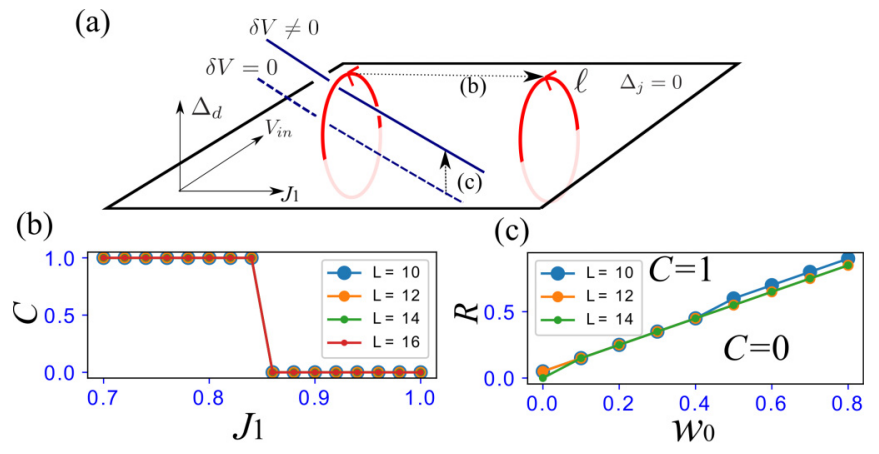

FIG. 5. (a) A schematic figure of the move of the pump loop as varying $J_{1}$ and the topological obstruction for a finite $\delta V$. Here, $\Delta_{d} \equiv$ $\Delta_{j=\text { odd }}-\Delta_{j=\text { even }} . \Delta_{d}$ is a symmetry-breaking parameter $\left(\lambda_{3}\right)$. The blue (dotted) line is a topological obstruction. (b) The behavior of $C_{N}$ of the (D1)-pump protocol as varying $J_{1}$. (c) The distribution of $C_{N}$ in the (D1)-pump protocol under the perturbation $\delta V$. For all results, there is almost no system size dependence.

in Fig. 5(c). As $R$ decreases, $C$ changes at a certain point, where the protocol loop intersects a gapless phase boundary. The TCP is robust for as long as the gapless phase boundary line (topological obstruction) exists within the protocol loop [see Fig. 5(a)].

Experimental realization. Our target model and pump protocol can be feasible by real experiments. In a cold atom optical lattice, the $\Delta_{j}$ term is fully controllable by adjusting a double-well optical lattice $[4,5]$. On the other hand, full control of the interactions has not yet been achieved in real experimental systems. However, the implementation of a controllable interaction is possible. For example, our target shape of the interaction can be implemented by selecting the kind of atom appropriately, such as a dipolar atom [56] (e.g., Cr [57], Er [58], and Dy [59]) and by fine tuning the spatial electric and/or magnetic external field patterns. Moreover, even if our interaction condition can be relaxed, $V_{j \in \text { odd }}=V_{\text {out }} \neq 0$, the iTCP persists [42]. This is an experimentally favorable situation.

Conclusion. Based on a simple Rice-Mele model extended by inclusion of an interaction, we have proposed the notion of an iTCP based on the topological phase transition of the SPT phase. A gapless phase boundary line works as a topological obstruction of the iTCP. Although the SPT phases with a gap closing phase transition are useful as a starting point, TCP is stable for the symmetry-breaking perturbation for as long as the gap along the pump is stable. We numerically demonstrated the presence of the iTCP, and also observed that the $\mathrm{BEC}$ is confirmed in the interacting case. Also, experimental pump protocols are proposed based on the iTCP.

Acknowledgments. This work is supported in part by JSPS KAKENHI Grant No. JP17H06138 (Y.K, Y.H.).
[1] D. J. Thouless, Phys. Rev. B 27, 6083 (1983).

[2] D. J. Thouless, M. Kohmoto, M. P. Nightingale, and M. den Nijs, Phys. Rev. Lett. 49, 405 (1982).
[3] Y. Hatsugai, Phys. Rev. Lett. 71, 3697 (1993).

[4] M. Lohse, C. Schweizer, O. Zilberberg, M. Aidelsburger, and I. Bloch, Nat. Phys. 12, 350 (2016). 
[5] S. Nakajima, T. Tomita, S. Taie, T. Ichinose, H. Ozawa, L. Wang, M. Troyer, and Y. Takahashi, Nat. Phys. 12, 296 (2016).

[6] C. Schweizer, M. Lohse, R. Citro, and I. Bloch, Phys. Rev. Lett. 117, 170405 (2016).

[7] Y. E. Kraus, Y. Lahini, Z. Ringel, M. Verbin, and O. Zilberberg, Phys. Rev. Lett. 109, 106402 (2012).

[8] T. Ozawa, H. M. Price, A. Amo, N. Goldman, M. Hafezi, L. Lu, M. C. Rechtsman, D. Schuster, J. Simon, O. Zilberberg, and I. Carusotto, Rev. Mod. Phys. 91, 015006 (2019).

[9] N. R. Cooper, J. Dalibard, and I. B. Spielman, Rev. Mod. Phys. 91, 015005 (2019).

[10] Y. Hatsugai and T. Fukui, Phys. Rev. B 94, 041102(R) (2016).

[11] M. Nakagawa, T. Yoshida, R. Peters, and N. Kawakami, Phys. Rev. B 98, 115147 (2018).

[12] T. Yoshida, R. Peters, S. Fujimoto, and N. Kawakami, Phys. Rev. Lett. 112, 196404 (2014).

[13] R. Li and M. Fleischhauer, Phys. Rev. B 96, 085444 (2017).

[14] Y. Ke, X. Qin, Y. S. Kivshar, and C. Lee, Phys. Rev. A 95, 063630 (2017).

[15] Y. Kuno, K. Shimizu, and I. Ichinose, New J. Phys. 19, 123025 (2017).

[16] A. Hayward, C. Schweizer, M. Lohse, M. Aidelsburger, and F. Heidrich-Meisner, Phys. Rev. B 98, 245148 (2018).

[17] S. Greschner, S. Mondal, and T. Mishra, Phys. Rev. A 101, 053630 (2020).

[18] F. Mei, G. Chen, N. Goldman, L. Xiao, and S. Jia, New J. Phys. 21, 095002 (2019).

[19] Y. Kuno, Phys. Rev. B 100, 054108 (2019).

[20] M. Ippoliti and R. N. Bhatt, Phys. Rev. Lett. 124, 086602 (2020).

[21] L. Privitera, A. Russomanno, R. Citro, and G. E. Santoro, Phys. Rev. Lett. 120, 106601 (2018).

[22] L. Zhou, D. Y. Tan, and J. Gong, Phys. Rev. B 92, 245409 (2015).

[23] H. Wang, L. Zhou, and J. Gong, Phys. Rev. B 91, 085420 (2015).

[24] Y. Hatsugai, J. Phys. Soc. Jpn. 75, 123601 (2006).

[25] F. Pollmann, A. M. Turner, E. Berg, and M. Oshikawa, Phys. Rev. B 81, 064439 (2010).

[26] X. Chen, Z.-C. Gu, Z.-X. Liu, and X.-G. Wen, Phys. Rev. B 87, 155114 (2013).

[27] A. Bermudez, E. Tirrito, M. Rizzi, M. Lewenstein, and S. Hands, Ann. Phys. 399, 149 (2018).

[28] Y. Hatsugai and I. Maruyama, Europhys. Lett. 95, 20003 (2011).

[29] T. Kariyado, T. Morimoto, and Y. Hatsugai, Phys. Rev. Lett. 120, 247202 (2018).

[30] Note that the symmetry is preserved with finite $\theta$.

[31] This circle $S^{1}$ combined with the pump loop defines a torus that is used to define a topological invariant for the iTCP.

[32] On the SPT plane, the energy gaps have to vanish on any path connecting the two points $P_{1}$ and $P_{2}$ since the quantized Berry phase $\gamma$ is stable and unchanged for as long as the gap remains open. Then this gap vanishing point forms a line (by changing the path) which is the gapless phase boundary as shown in Fig. 1.
[33] Similar situations are discussed for the TCP in an extended Bose-Hubbard model in previous studies: E. Berg, M. Levin, and E. Altman, Phys. Rev. Lett. 106, 110405 (2011); D. Rossini, M. Gibertini, V. Giovannetti, and R. Fazio, Phys. Rev. B 87, 085131 (2013).

[34] S. Ryu and Y. Hatsugai, Phys. Rev. Lett. 89, 077002 (2002).

[35] Y. Hatsugai, Solid State Commun. 149, 1061 (2009).

[36] The bond-centered inversion transformation is defined by $c_{j} \rightarrow$ $c_{L-j-1}$.

[37] M. J. Rice and E. J. Mele, Phys. Rev. Lett. 49, 1455 (1982).

[38] J. K. Asboth, L. Oroszlany, and A. Palyi, A Short Course on Topological Insulators: Band-structure Topology and Edge States in One and Two Dimensions (Springer, Berlin, 2016).

[39] W. P. Su, J. R. Schrieffer, and A. J. Heeger, Phys. Rev. Lett. 42, 1698 (1979).

[40] Y. Kuno, Phys. Rev. B 99, 064105 (2019).

[41] S. Aoki, Phys. Rev. D 30, 2653 (1984).

[42] See Supplemental Material at http://link.aps.org/supplemental/ 10.1103/PhysRevResearch.2.042024 for mean-field studies and effects of both inner- and inter-unit-cell interactions.

[43] Y. Araki and T. Kimura, Phys. Rev. B 87, 205440 (2013); Y. Araki, T. Kimura, A. Sekine, K. Nomura, and T. Z. Nakano, arXiv:1311.3973.

[44] Y. Hatsugai, J. Phys.: Condens. Matter 19, 145209 (2007).

[45] H. Guo, S.-Q. Shen, and S. Feng, Phys. Rev. B 86, 085124 (2012).

[46] $\gamma$ is measured in units of $2 \pi$.

[47] We may construct a nontrivial iTCP enclosing the CDW (Aoki) phase which is continuously connected to the gap closing line. In this sense, the symmetry-breaking phase also works as a topological obstruction of the nontrivial iTCP.

[48] Q. Niu, D. J. Thouless, and Y.-S. Wu, Phys. Rev. B 31, 3372 (1985).

[49] K. Kudo, H. Watanabe, T. Kariyado, and Y. Hatsugai, Phys. Rev. Lett. 122, 146601 (2019).

[50] U. Schollwock, Ann. Phys. (Amsterdam) 326, 96 (2011).

[51] J. Hauschild and F. Pollmann, SciPost Phys. Lect. Notes, 5 (2018), doi: 10.21468/SciPostPhysLectNotes.5.

[52] The phase boundary was also determined by the behavior of the entanglement entropy and the behavior of the center of mass under a very small inversion-breaking potential.

[53] H. Hu, C. Cheng, Z. Xu, H. G. Luo, and S. Chen, Phys. Rev. B 90, 035150 (2014).

[54] Consider an energy of a many-body state with particle $N_{e}$, $E_{N_{e}}$. Then $N_{e}$ of the grand canonical ground state needs to satisfy $E_{N_{e}+1}-\mu\left(N_{e}+1\right)>0$ and $E_{N_{e}}-\mu N_{e}<0$. It implies $\Delta E\left(N_{e}, t\right)<\mu$.

[55] T. Fukui, Y. Hatsugai, and H. Suzuki, J. Phys. Soc. Jpn. 74, 1674 (2005).

[56] T. Lahaye, C. Menotti, L. Santos, M. Lewenstein, and T. Pfau, Rep. Prog. Phys. 72, 126401 (2009).

[57] A. de Paz, A. Sharma, A. Chotia, E. Marechal, J. H. Huckans, P. Pedri, L. Santos, O. Gorceix, L. Vernac, and B. Laburthe-Tolra, Phys. Rev. Lett. 111, 185305 (2013).

[58] S. Baier, M. J. Mark, D. Petter, K. Aikawa, L. Chomaz, Z. Cai, M. Baranov, P. Zoller, and F. Ferlaino, Science 352, 201 (2016).

[59] M. Lu, N. Q. Burdick, and B. L. Lev, Phys. Rev. Lett. 108, 215301 (2012). 